\title{
Journal of

\section{Structural Studies on Dinuclear Ruthenium(II) Complexes That Bind Diastereoselectively to an Antiparallel Folded Human Telomere Sequence}

\author{
Tom Wilson, ${ }^{\dagger, \ddagger}$ Paulo J. Costa, ${ }^{\S}$ Vítor Félix, $^{*, \S}$ Mike P. Williamson, ${ }^{*}{ }^{\ddagger}$ and Jim A. Thomas ${ }^{* \dagger}$ \\ ${ }^{\dagger}$ Department of Chemistry, University of Sheffield, Dainton Building, Brook Hill, Sheffield S3 7HF, U.K. \\ "Department of Molecular Biology and Biotechnology, University of Sheffield, Firth Court, Western Bank, Sheffield S10 2TN, U.K. \\ ${ }^{\S}$ Departamento de Química, CICECO and Secção Autónoma de Ciências da Saúde, Universidade de Aveiro, 3810-193 Aveiro, \\ Portugal
}

Supporting Information

ABSTRACT: We report DNA binding studies of the dinuclear ruthenium ligand $\left[\left\{\mathrm{Ru}(\mathrm{phen})_{2}\right\}_{2} \mathrm{tpphz}\right]^{4+}$ in enantiomerically pure forms. As expected from previous studies of related complexes, both isomers bind with similar affinity to BDNA and have enhanced luminescence. However, when tested against the G-quadruplex from human telomeres (which we show to form an antiparallel basket structure with a diagonal loop across one end), the $\Lambda \Lambda$ isomer binds approximately 40 times more tightly than the $\Delta \Delta$, with a stronger luminescence.

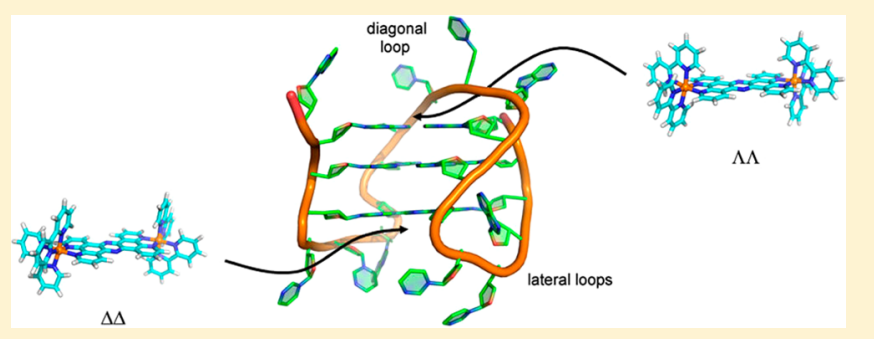
NMR studies show that the complex binds at both ends of the quadruplex. Modeling studies, based on experimentally derived restraints obtained for the closely related $\left[\left\{\mathrm{Ru}(\mathrm{bipy})_{2}\right\}_{2} \mathrm{tpphz}\right]^{4+}$, show that the $\Lambda \Lambda$ isomer fits neatly under the diagonal loop, whereas the $\Delta \Delta$ isomer is unable to bind here and binds at the lateral loop end. Molecular dynamics simulations show that the $\Delta \Delta$ isomer is prevented from binding under the diagonal loop by the rigidity of the loop. We thus present a novel enantioselective binding substrate for antiparallel basket G-quadruplexes, with features that make it a useful tool for quadruplex studies.

\section{INTRODUCTION}

Although it has been known for five decades that guanine-rich nucleic acids can form four-stranded structures, research into quadruplex DNA has rapidly escalated in recent years. One reason for continued interest is the demonstration that telomeres can and do fold into quadruplex structures in vivo. ${ }^{1-3}$ Shortening of telomeres on chromosomal replication is considered to be a major cause of senescence, and cancer cells have been shown to generate an immortal phenotype by upregulating telomerase., ${ }^{4,5}$ The activity of telomerase is inhibited by the presence of G-quadruplexes, ${ }^{6}$ leading to the possibility of novel anticancer agents that work by binding to and stabilizing such quadruplexes.

A second reason for interest is the observation that quadruplexes are found not only in telomeres but also in other parts of the genome. Typically they are found in upstream promoters ${ }^{7-9}$ and in some cases have been shown to perform a regulatory function on downstream genes. ${ }^{10-13}$ Quadruplexes are also formed by RNA and again are likely to have regulatory roles on translation. ${ }^{14}$ For all these reasons, there is considerable interest in finding small molecules that bind to quadruplexes and stabilize them and that could act as markers for their presence.

Over the past few years, it has become abundantly clear that guanine-rich sequences can fold into quadruplexes in many different ways. $^{15}$ A given sequence can also fold differently depending on solution conditions, including counterions (potassium or sodium), molecular crowding, ${ }^{16-18}$ and dehydration. ${ }^{19} \mathrm{~A}$ case in point is the human telomere sequence, HTS, $\mathrm{d}\left[\mathrm{AG}_{3}\left(\mathrm{TTAG}_{3}\right)_{3}\right]$, which has been observed in several conformations. $^{20-27}$ It appears that such behavior is common. ${ }^{28}$ This plasticity makes it all the more important to identify small molecules that bind specifically to particular conformations and stabilize them, especially if the function and dysfunction of quadruplexes in normal and abnormal cellular function are to be delineated. ${ }^{29}$

Despite this importance, there is little detailed crystallographic or NMR data on ligand-HTS quadruplex structures. $^{28,30,31}$ Of relevance to this work, there is only one report on metal complexes. ${ }^{32}$ Only four X-ray structures involving an intramolecular quadruplex have been reported, all of which involve the all-parallel conformer with the ligand end-stacking on terminal G-tetrads. ${ }^{33-36}$ Although, as outlined above, telomere sequences can take up a range of topologies, virtually all the other reported structures also involve ligands bound to all-parallel conformers, comprising tetramolecular or bimolecular quadruplexes. ${ }^{37-41}$ Indeed, given this paucity of data and

Received: July 24, 2013

Published: October 2, 2013 
A

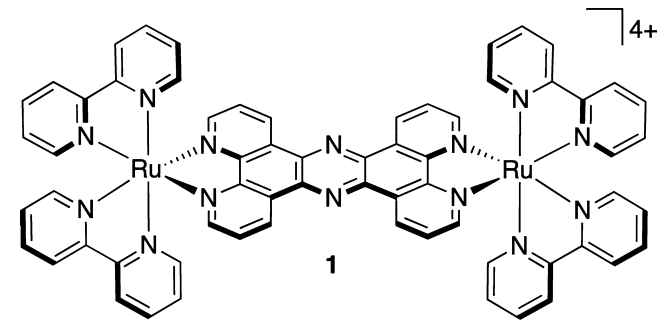

B

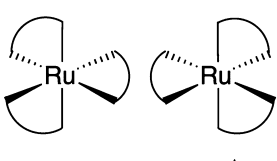

C
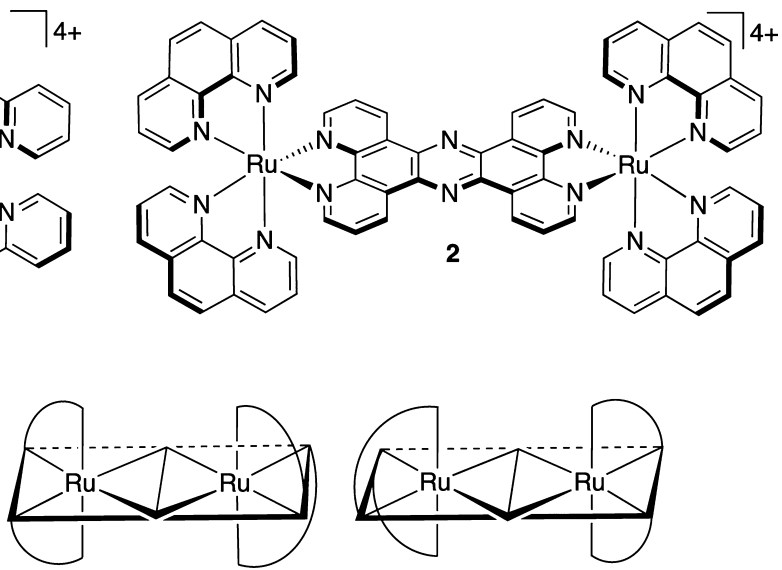

Figure 1. (A) Structures of complexes studied. (B) The two possible enantiomers of each of the metal centers in $\mathbf{1}$ and 2. (C) Schematic of the two diastereomers of 1 and 2 relevant to this study: left $\Lambda \Lambda$; right $\Delta \Delta$.

the range of potential telomeric conformer targets, it has been suggested that the design of small molecules to stabilize Gquadruplexes should also be directed toward ligands that selectively target antiparallel and hybrid type G-quadruplex folding topologies. ${ }^{27}$ The structural data obtained for small molecules bound to non all-parallel quadruplex conformers indicate that these telomeric structures could be targeted through specific interactions. For example the crystallographic structures of disubstituted aminoalkylamidoacridine derivatives bound to the dimeric antiparallel G-quadruplex formed from the Oxytricha nova telomere sequence $\mathrm{d}\left(\mathrm{G}_{4} \mathrm{~T}_{4} \mathrm{G}_{4}\right)$ reveal that while these structures display the expected end-stacking interaction, they also feature a second distinctive motif: the acridine moiety "threads" through the $\mathrm{T}_{4}$ diagonal loop. ${ }^{42,43}$

As part of a program to develop luminescent metal complexes as sequence and structure specific DNA binding substrates, $^{44-46}$ we have studied the quadruplex binding properties of dinuclear ruthenium(II) complexes containing

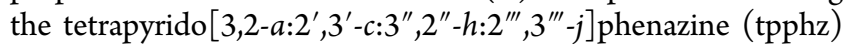
ligand. Although the central tpphz ligand in such complexes is planar, the octahedral coordination geometry about the ruthenium centers gives rise to a "dumbbell" structure with bulky, and chiral, "stoppers". These studies have revealed that both $\left[\left\{\mathrm{Ru}(\text { bipy })_{2}\right\}_{2} \text { tpphz }\right]^{4+}, \mathbf{1}$, and $\left[\left\{\mathrm{Ru}(\text { phen })_{2}\right\}_{2} \mathrm{tpphz}\right]^{4+}, \mathbf{2}$, (where bipy $=2,2^{\prime}$-bipyridine, phen $=1,10$-phenanthroline), Figure 1, bind to quadruplex DNA with high affinities $\left(>10^{7}\right.$ $\left.\mathrm{M}^{-1}\right)$.

Both these complexes display a "DNA light-switch" effect, being essentially nonemissive in aqueous solution until DNA binding induces a several orders of magnitude increase in their $\mathrm{Ru} \rightarrow \operatorname{tpphz}{ }^{3}$ MLCT-based luminescence. Uniquely, the emission and binding affinities of $\mathbf{1}$ and $\mathbf{2}$ are sensitive to DNA structure. While groove binding to all duplexes produces a relatively weak emission at $>675 \mathrm{~nm}$, binding to quadruplexes produces more complex emission changes. Intense blue-shifted luminescence $(\sim 630 \mathrm{~nm})$ and high affinity binding is observed only when the complex binds to antiparallel quadruplex structures containing external diagonal loops at least three bases in length. The presence of shorter lateral loops limits binding affinities by several orders of magnitude and results in negligible emission. ${ }^{48}$ This difference in luminescence output means that, despite the only modest selectivity in binding affinities, quadruplex structures can be detected in the presence of duplex DNA. Indeed, this concept has been illustrated by recently reported cell studies with these complexes. ${ }^{49}$

Fascinatingly, in cellulo studies reveal that, while $\mathbf{1}$ is only taken up by fixed cells, $\mathbf{2}$ is actively transported into live cells. $^{49,50}$ Confocal microscopy studies confirm that $\mathbf{2}$ is a selective luminescent stain for heterochromatin. Furthermore, 2 displays distinctive noncolocalized multiple emission peaks, whose wavelengths are consistent with those obtained through in vitro studies, indicating that the complex is an in cellulo probe of DNA structure. ${ }^{49}$

Until now, these studies have used racemic mixtures of complexes $\mathbf{1}$ and $\mathbf{2}$. However, recently the $\mathrm{Qu}$ group have shown that the $\Lambda \Lambda$-enantiomer of a nonemissive dinuclear nickel(II) triple helicate complex displays a strong binding preference for specific quadruplex structures over duplex DNA, ${ }^{51,52}$ while Sugiyama and co-workers have demonstrated that a chiral helicene macrocycle can enantioselectively recognize quadruplex DNA. ${ }^{53}$ Furthermore, recent spectroscopic, ${ }^{54,55}$ crystallographic, ${ }^{56-58}$ and $\mathrm{NMR}^{59}$ studies on monoand dinuclear $\mathrm{Ru}^{\mathrm{II}}(\mathrm{dppz})\left(\mathrm{dppz}=\operatorname{dipyrido}\left[3,2-a: 2^{\prime}, 3^{\prime}-c\right]\right.$ phenazine) systems with duplex DNA have also illustrated the importance of chirality in such interactions. In light of these studies, we discuss the DNA binding preferences of enantiopure samples of $\mathbf{2}$ and also report NMR-based studies designed to delineate the structural details of quadruplex binding by 1 and 2 , followed by further rationalization of the results based on molecular dynamics (MD) simulations in water.

\section{RESULTS AND DISCUSSION}

Spectroscopic Binding Studies. In previous studies, we have found that the luminescent binding response of $\mathrm{rac}-1$ and rac-2 to duplex and quadruplex DNA is effectively identical. In both cases, binding to duplex DNA produces a 60-fold increase in emission, while binding to quadruplex produces a $>150$ times increase in blue-shifted emission. Furthermore comparisons of $K_{\mathrm{b}}$ values for $\mathrm{rac}-\mathbf{1}$ and $\mathrm{rac}-\mathbf{2}$ revealed they are almost identical within experimental error. ${ }^{47}$ In the study reported herein, the interaction of enantiomerically pure complexes $\Delta \Delta-2$ and $\Lambda \Lambda$ 2 with duplex and quadruplex DNA was investigated through luminescence titrations using calf thymus DNA (CT-DNA) (Supporting Information Figure S01) and the human telomere sequence $(\mathrm{HTS}) \mathrm{d}\left[\mathrm{AG}_{3}\left(\mathrm{TTAG}_{3}\right)_{3}\right]$ (Figure 2). 


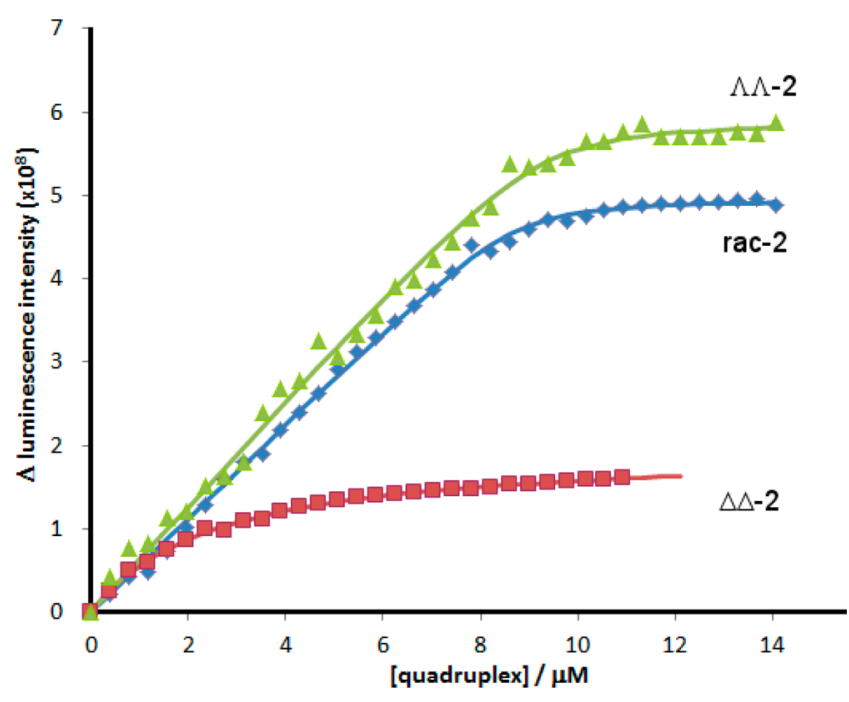

Figure 2. Typical data for the luminescence response of $\Lambda \Lambda-2, \Delta \Delta-2$, and an unresolved diastereomeric mixture of 2 to the progressive addition of the unimolecular HTS quadruplex $\mathrm{d}\left[\mathrm{AG}_{3}\left(\mathrm{TTAG}_{3}\right)_{3}\right]$. Conditions: $10 \mathrm{mM} \mathrm{KH} \mathrm{PO}_{4} / \mathrm{K}_{2} \mathrm{HPO}_{4}, 1 \mathrm{mM} \mathrm{K}{ }_{2} \mathrm{EDTA}, 200 \mathrm{mM}$ $\mathrm{KCl}, \mathrm{pH} 7.0,298 \mathrm{~K},[$ complex $]=7 \mu \mathrm{M}$. Lines show fitted $K_{\mathrm{b}}$.

To aid comparisons, data for $\mathrm{rac}-2$ in these conditions are also included. The CD spectrum of the HTS sequence in uncrowded $\mathrm{K}^{+}$solutions confirmed that it adopts an antiparallel basket conformation (Supporting Information Figure S02), which is consistent with previous observations by Renčiuk et $\mathrm{al}^{26} \mathrm{NMR}$ and CD spectra of HTS in $100 \mathrm{mM} \mathrm{NaCl}$ are similar to those reported by Wang and Patel ${ }^{20}$ and indicate that the structure remains an antiparallel basket.

Characteristics for the interaction of CT-DNA with either enantiomer in aqueous buffer solutions were found to be very similar. Both show a very similar increase in steady-state luminescence, which is identical within experimental error to the changes observed for an unresolved diastereomeric mixture (see Supporting Information). Indeed, fits of the data to standard binding models lead to estimates of binding affinities that are almost identical to those previously reported for the unresolved mixture $\left(K_{\mathrm{b}}=4.40 \times 10^{6} \mathrm{M}^{-1}\right)$, although it does appear that the binding affinity of $\Lambda \Lambda-2$ is slightly higher than that of $\Delta \Delta-2$ (Table 1$)$.

Table 1. Estimated Binding Affinities for Different Diastereomers of 2 with Duplex and Quadruplex DNA ${ }^{a}$

\begin{tabular}{lcc}
\multicolumn{1}{c}{ complex } & $K_{\mathrm{b}}(\mathrm{CT}-\mathrm{DNA}) ; M^{-1}$ & $K_{\mathrm{b}}(\mathrm{HTS}) ; \mathrm{M}^{-1}$ \\
$\Lambda \Lambda$-2 & $6.73 \times 10^{6}$ & $1.16 \times 10^{7}$ \\
$\Delta \Delta-2$ & $1.99 \times 10^{6}$ & $2.95 \times 10^{5}$ \\
${\text { unresolved } 2^{b}}^{b}$ & $6.68 \times 10^{6}$ & $1.77 \times 10^{7}$
\end{tabular}

${ }^{a}$ Calculated errors in estimates of $K_{\mathrm{b}} \approx \pm 20 \%$. ${ }^{b}$ Binding constants for diastereomeric mixture are apparent $K_{\mathrm{b}} \mathrm{s}$.

By contrast, titrations of enantiomerically pure 2 with HTS produced clear differences in the luminescent response of $\Lambda \Lambda-2$ and $\Delta \Delta-2$. Addition of HTS to $\Lambda \Lambda-2$ led to increases in emission that were around $20 \%$ larger than those observed for an unresolved diastereomeric mixture. More fascinatingly, $\Delta \Delta$ 2 displayed a much smaller emission response than either $\Lambda \Lambda-2$ or the unresolved solution mixture: at binding saturation, the steady-state emission intensity of $\Delta \Delta-2$ is 6-fold less than that of $\Lambda \Lambda-2$ (Figure 2). Because the binding-induced light-switch effect observed for these complexes is due to transfer from a polar bulk aqueous environment into a less polar binding environment, these data indicate that bound $\Delta \Delta-2$ is much more solvent accessible than $\Lambda \Lambda-2$, thus implying structural differences in the binding complexes with HTS for the two diastereomers. Fits of the luminescence changes to a simple one set of identical binding sites model offers further evidence to support this hypothesis, as the binding affinity of $\Lambda \Lambda-2$ is around 40 times higher than that of $\Delta \Delta-2$ (Table 1). The diastereomeric mixture contains $25 \% \Lambda \Lambda-2,25 \% \Delta \Delta-2$, and $50 \% \Lambda \Delta-2$, yet its emission intensity is roughly $85 \%$ the intensity of pure $\Lambda \Lambda-2$. This is almost exactly the value expected if $\Lambda \Delta-2$ has the same emission intensity as $\Lambda \Lambda-2$. The results therefore suggest that $\Lambda \Delta-2$ binds in the same way as $\Lambda \Lambda-2$, i.e., that for binding to HTS, $\Delta$ chirality is possible at one end of the ligand but not both.

As discussed before, addition of $\mathbf{2}$ (as a racemic mixture or as pure enantiomers) gives rise to hypochromic and bathochromic shifts in UV-vis spectra, indicative of stacking of the aromatic rings against DNA base pairs. A stacking mode of binding is also indicated by the strongly enhanced luminescence and the blue-shift of approximately $30 \mathrm{~nm}$, which we have shown only occurs when the ligand is strongly shielded from solvent. ${ }^{48}$ Shielding to this extent can only arise when the ligand is covered by quadruplex loops: in other words, it implies stacking over a tetrad plane and shielding by loops, rather than groove binding. Furthermore, the markedly greater increase in luminescence for bound $\Lambda \Lambda-2$ implies significantly better shielding from solvent for this isomer. Further evidence for this hypothesis was obtained by NMR studies

NMR Binding Studies. To provide structural insights into the effects observed in our optical studies, the binding of $\mathbf{1}$ and 2 to HTS was further investigated using a combination of NMR spectroscopy and simulated annealing coupled with restrained molecular dynamics simulations for structure determination. Assignments of the folded quadruplex before addition of any complex are given in the Supporting Information (Table S1).

On addition of $\Delta \Delta-2$ to HTS, severe line broadening of DNA signals was observed by NMR, mainly from the lateral loop end (red dots, bottom end of Figure 3). This implies that the ligand is binding at this end and causing structural perturbations concomitant with binding, which occur on a time scale in the millisecond range. Unfortunately, this is a common observation in studies of DNA/ligand interactions and it makes structure determination of the complexes difficult by severely reducing the information content of spectra. By contrast, addition of the more tightly binding $\Lambda \Lambda-2$ produced less severe relaxation-induced broadening although enough to abolish intermolecular NOEs. Chemical shift changes occurred at both ends of the quadruplex, and intramolecular NOEs were broadened and lost at both ends. It therefore appears that whereas $\Delta \Delta-2$ binds mainly at the lateral loop end, $\Lambda \Lambda-2$ binds at both ends. Taken together with the luminescence data, the implication is that binding of $\Lambda \Lambda-2$ at the diagonal loop end (top end of Figure 3) is accompanied by a high degree of shielding of the ligand from solvent. This in turn implies that the ligand is stacked onto the tetrad underneath the diagonal loop.

All attempts to alter the solution conditions so as to bring back intermolecular NOEs were unsuccessful. We therefore carried out titrations with 1 . The absorption and emission response of this complex to HTS binding is very similar to that of 2: in particular, the enhancement and blue-shifting of its 


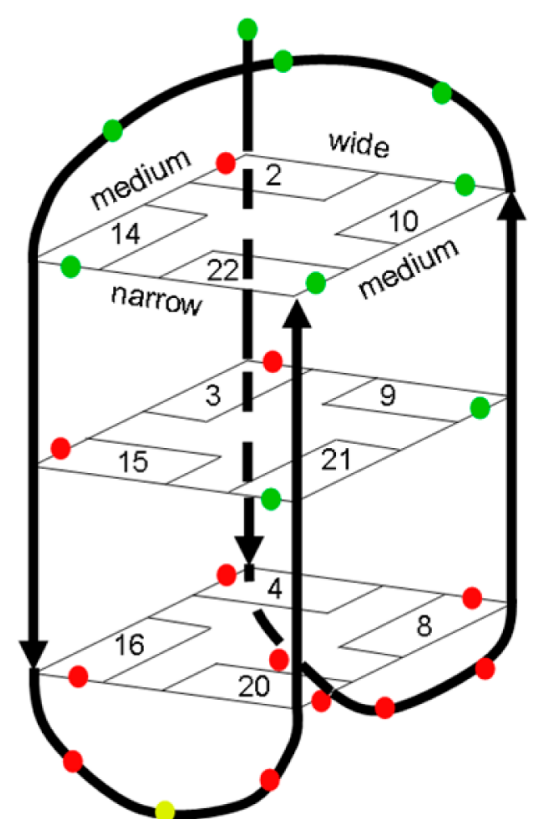

Figure 3. The antiparallel basket formed by HTS. The colors show the residues still present (green), missing (red), and possible exchange doublet (yellow), upon addition of $\Delta \Delta-\mathbf{2}$.

luminescence are very similar, indicating analogous modes of binding. ${ }^{48}$ These studies were carried out on a diastereomeric mixture as this had the advantage that both putative binding sites could be potentially investigated in a single experiment.

Addition of 1 to HTS gave rise to chemical shift changes at both ends of the quadruplex. There was broadening of signals throughout, which led to a general reduction in the intensities of intramolecular NOEs, although the largest effects were seen at the lateral loop end (Table 2). However, gratifyingly, a large number of new intermolecular NOEs could be seen to the ligand (Figure 4).

In free solution, NMR resonances from the four symmetryrelated positions of $\mathbf{1}$ have identical chemical shifts. However, in the presence of HTS, most signals were split into four, in some cases with fairly large chemical shift changes. The increased complexity made it impossible to assign the ligand signals in the complex to individual positions and meant that, although we were able to observe 20 intermolecular NOEs in the complex, we were only able to assign the nucleotide signals (Table 2).

Analysis of the NOEs reveals that they are not compatible with a single structure for the complex, because 13 derive from contacts at the diagonal loop end and seven from contacts at the lateral loop end. On the basis of the discussion above, this is not surprising. In particular, by analogy with 2 , we expect $\Delta \Delta-1$ to bind mainly at the lateral loop end and $\Lambda \Lambda-1$ (and probably also $\Lambda \Delta-1)$ mainly at the diagonal loop end. NOE intensities are compatible with this expectation. As anticipated, the NOEs at the diagonal loop end are consistent with 1 binding under the diagonal loop.

NMR-Based Structures of Complexes. The experimentally observed NOEs from the lateral loop end were used to calculate structures for both $\Lambda \Lambda-1$ and $\Delta \Delta-1$ bound at the lateral loop end using restrained simulated annealing. The structure generated for $\Delta \Delta-\mathbf{1}$ is shown in Figure 5 and has no violations of the NOE constraints greater than $0.5 \AA$.
Table 2. Intermolecular NOE Crosspeaks and the Corresponding DNA Residues Identified for the Interaction of 1 with HTS

$\begin{array}{ccc}\text { complex signal } & \begin{array}{c}\text { DNA signal } \\ \text { One-Loop End }\end{array} & \text { assigned DNA atom } \\ 7.79 & 4.38 & \mathrm{G} 10 \mathrm{H} 5^{\prime} \\ 7.79 & 2.89 & \mathrm{G} 14 \mathrm{H} 2^{\prime} \\ 7.79 & 2.74 & \mathrm{G} 14 \mathrm{H} 2^{\prime \prime} \\ 7.80 & 5.65 & \mathrm{~T} 11 \mathrm{H} 1^{\prime} \\ 7.80 & 6.30 & \mathrm{G} 10 \mathrm{H} 1^{\prime} \\ 7.76 & 6.31 & \mathrm{G} 10 \mathrm{H} 1^{\prime} \\ 7.74 & 3.31 & \mathrm{G} 22 \mathrm{H} 2^{\prime} \\ 7.55 & 2.40 & \mathrm{G} 22 \mathrm{H} 2^{\prime \prime} \\ 6.95 & 5.58 & \mathrm{~T} 12 \mathrm{H} 1^{\prime} \\ 6.95 & 1.14 & \mathrm{~T} 12 \mathrm{H} 2^{\prime} \\ 6.29 & 5.46 & \mathrm{G} 2 \mathrm{H} 1^{\prime} \\ 8.36 & 2.61 & \mathrm{G} 9 \mathrm{H} 2^{\prime \prime} \\ 8.36 & 2.94 & \mathrm{G} 9 \mathrm{H} 2^{\prime} \\ 8.36 & \text { Two-Loop End } & \\ 8.36 & 2.51 & \mathrm{G} 8 \mathrm{H} 2^{\prime \prime} \\ 8.11 & 3.08 & \mathrm{G} 8 \mathrm{H} 2^{\prime} \\ 6.93 & 1.91 & \mathrm{~T} 5 \mathrm{CH} 3 \\ 6.93 & 1.30 & \mathrm{~T} 18 \mathrm{H} 2^{\prime} \\ 6.93 & 2.07 & \mathrm{~T} 17 \mathrm{CH} 3 \\ 8.22 & 4.04 & \mathrm{~A} 19 \mathrm{H}^{\prime}{ }^{\prime} \\ & 2.83 & \end{array}$

While the tpphz ligand stacks on top of the tetrad bases, this interaction is reinforced by electrostatics: the positively charged ruthenium centers are located at the edge of the tetrad close to the anionic phosphate backbone. There is little perturbation to the quadruplex structure, with slight movement of the lateral loops to accommodate the ligand. Bases in the loops partially shield the tpphz rings, as expected from the luminescence. There is little direct contact between the bipy ligands and the quadruplex, and therefore both the $\Lambda \Lambda$ and $\Delta \Delta$ complexes bind in a similar way, with similar energies.

The observed NOEs were also used to calculate a structure for the complex bound at the diagonal loop end. The bound $\Lambda \Lambda-1$ structure is shown in Figure 6 . The bipy rings fit neatly against the phosphate backbone making close van der Waals contact. The diagonal loop holds the tpphz in place and shields it from solvent, as expected from the luminescence data.

By contrast, attempts to use the same NOEs to calculate a structure with $\Delta \Delta-\mathbf{1}$ bound at the same site result in a very high-energy state (roughly 10 times higher). In this structure, one end of the ligand is able to fit straightforwardly, by displacing the terminal nucleotide of the quadruplex DNA chain (Figure 7). However, in this calculation, the other end of the ligand does not displace the DNA backbone. Instead, the calculation generates a number of physically impossible solutions, of which the lowest energy is shown in Figure 7: the phosphate backbone passes through the middle of one of the aromatic bipy rings. The other solution is to displace one bipy ligand completely away from the tpphz plane. Structures have been calculated for the $\Lambda \Lambda$ and $\Delta \Delta$ isomers of 2 in the same way and show similar features (Supporting Information Figures S04 and S05). These calculations imply that the experimental NOE data are compatible with the $\Lambda \Lambda$ isomer binding at the diagonal loop end but not with the $\Delta \Delta$ isomer binding at the same site. This result is thus in agreement with the conclusion reached above, that both isomers bind at the 


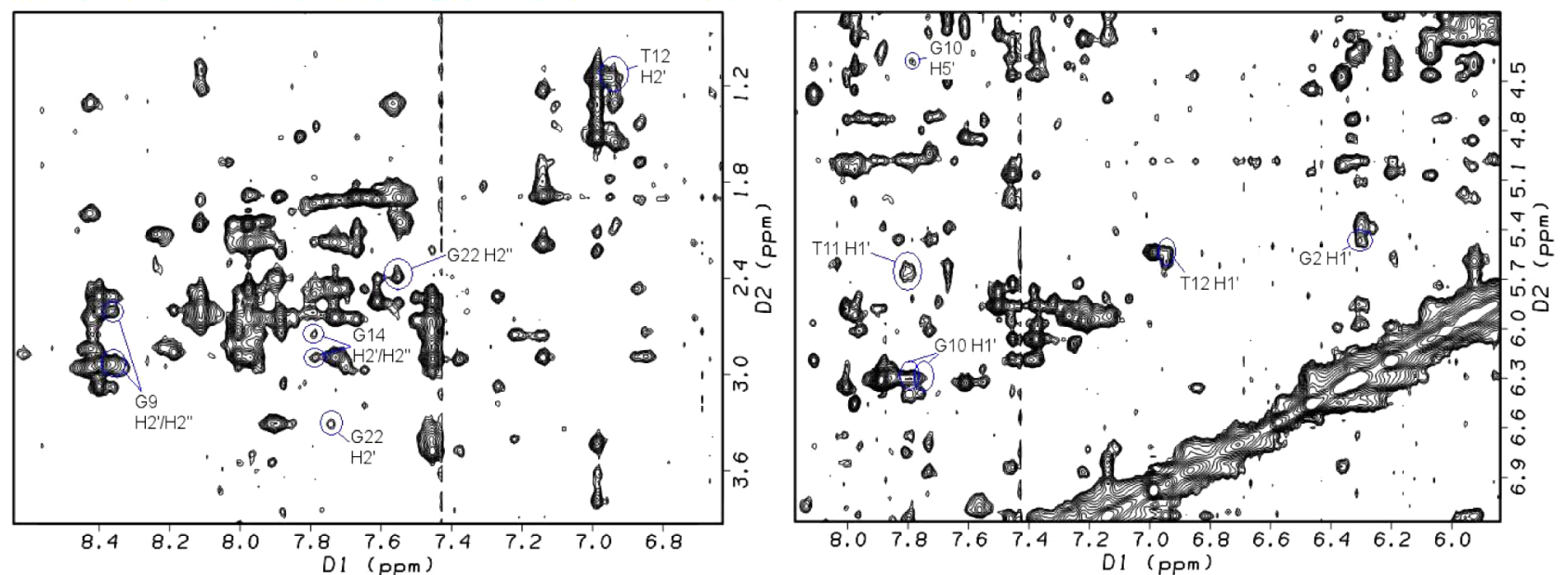

Figure 4. Selected sections of spectra showing the new crosspeaks identified upon addition of a diastereomeric mixture of $\mathbf{1}$ to the HTS sequence.

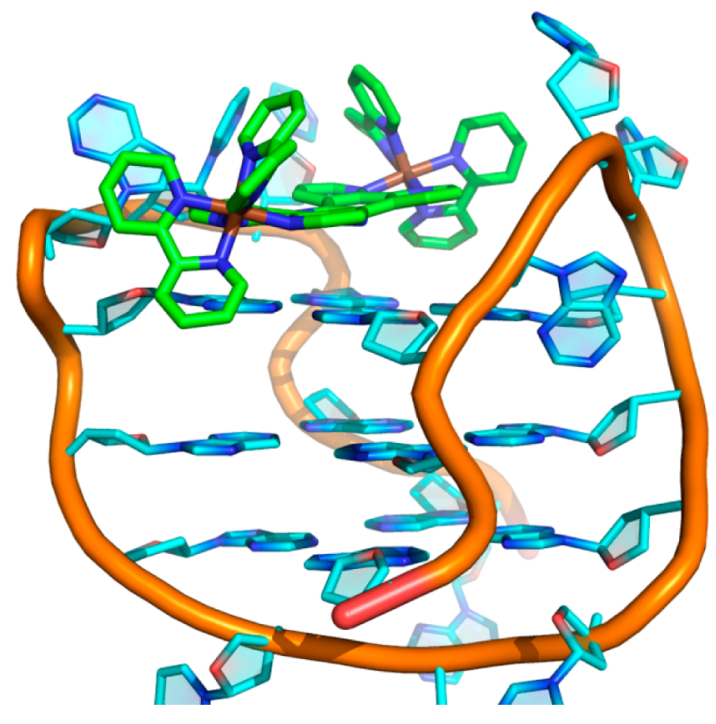

Figure 5. Molecular dynamics-based structure of $\Delta \Delta-1$ bound to the lateral loop end of HTS, generated using experimentally observed NOEs for the interaction.
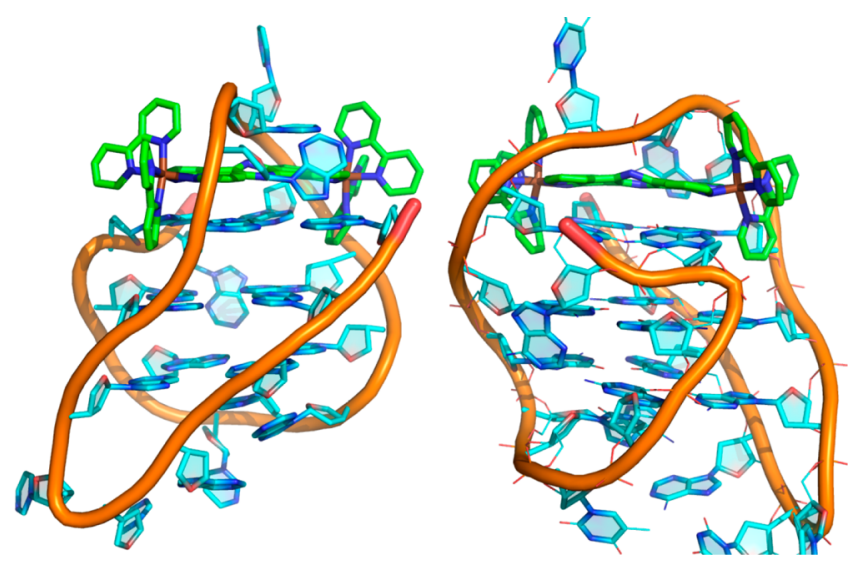

Figure 6. Molecular dynamics-based structure of $\Lambda \Lambda-1$ bound to the diagonal loop end of HTS, generated using experimentally observed NOEs for the interaction. Left: Illustrating the bipy ring at front left parallel to the DNA backbone. Right: Showing how the $5^{\prime}$ end of the DNA backbone (front center) moves to avoid the ligand.

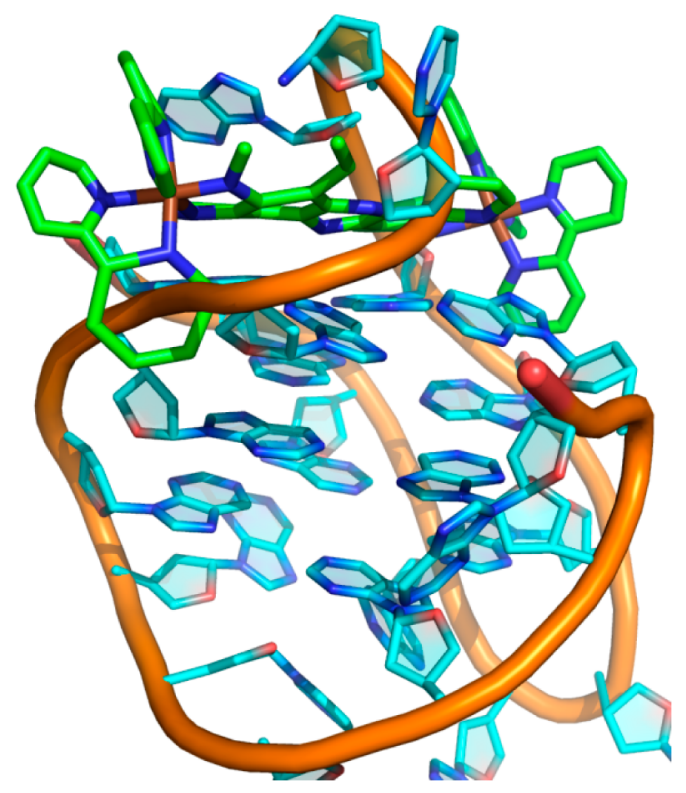

Figure 7. Molecular dynamics-based structure of $\Delta \Delta-1$ bound to the diagonal loop end of HTS, generated using experimentally observed NOEs for the interaction.

lateral loop end, but only the $\Lambda \Lambda$ isomer (and probably the $\Lambda \Delta$ isomer also) binds at the diagonal loop end. To investigate this issue further, unconstrained molecular dynamics simulations were carried out in a TIP3P water model.

Unconstrained MD Simulations. To evaluate the impact of $\Lambda \Lambda-1$ and $\Delta \Delta-1$ complexes on the quadruplex structure when binding under the diagonal loop, molecular dynamics simulations were performed on free HTS and its association with $\Lambda \Lambda-1$ and $\Delta \Delta-\mathbf{1}$. For free HTS, three independent simulations were undertaken using the structures presented in Figures 5-7 (henceforth denoted A, B, and C) as starting conformations. These results were then compared with simulations of the associations formed between the quadruplex and both diastereomers $\Lambda \Lambda-1$ and $\Delta \Delta-\mathbf{1}$, labeled $\Lambda \Lambda$-1-HTS and $\Delta \Delta-1-H T S$, respectively. For the $\Lambda \Lambda$-1-HTS simulation, the NMR-based structure shown in Figure 6 was used as starting point, while for simulation of $\Delta \Delta-1$-HTS, the starting structure was generated from $\mathbf{\Lambda} \mathbf{\Lambda}-\mathbf{1}-\mathrm{HTS}$ by replacing $\Lambda \Lambda-\mathbf{1}$ with $\Delta \Delta-1$, superimposing the two ruthenium atoms and the 

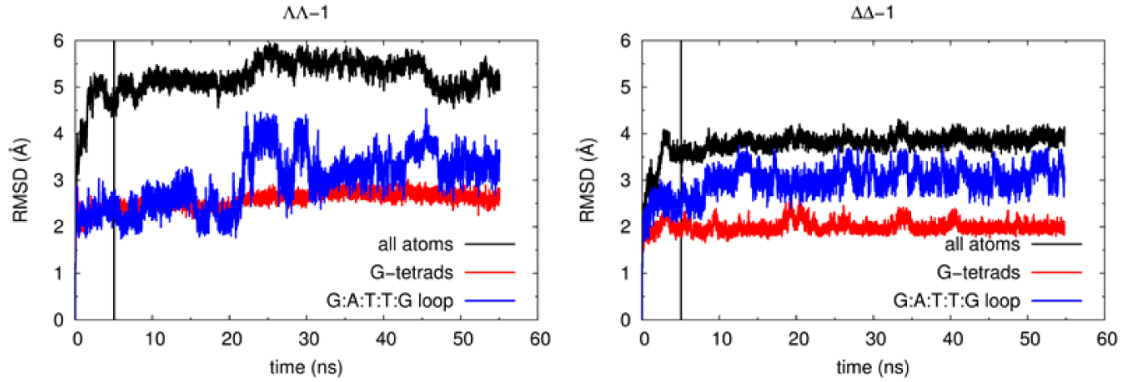

Figure 8. Variation of the RMSD values throughout the course of the MD simulations for $\mathbf{\Lambda} \mathbf{\Lambda}$-1-HTS (left) and $\boldsymbol{\Delta} \boldsymbol{\Delta}$-1-HTS (right). The vertical black line marks the separation between the equilibration and collection simulation stages.
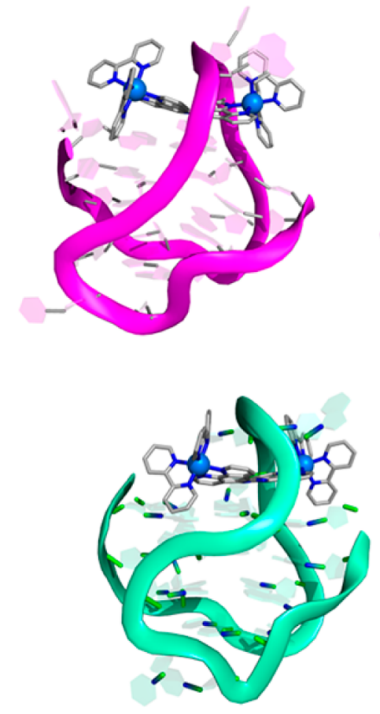
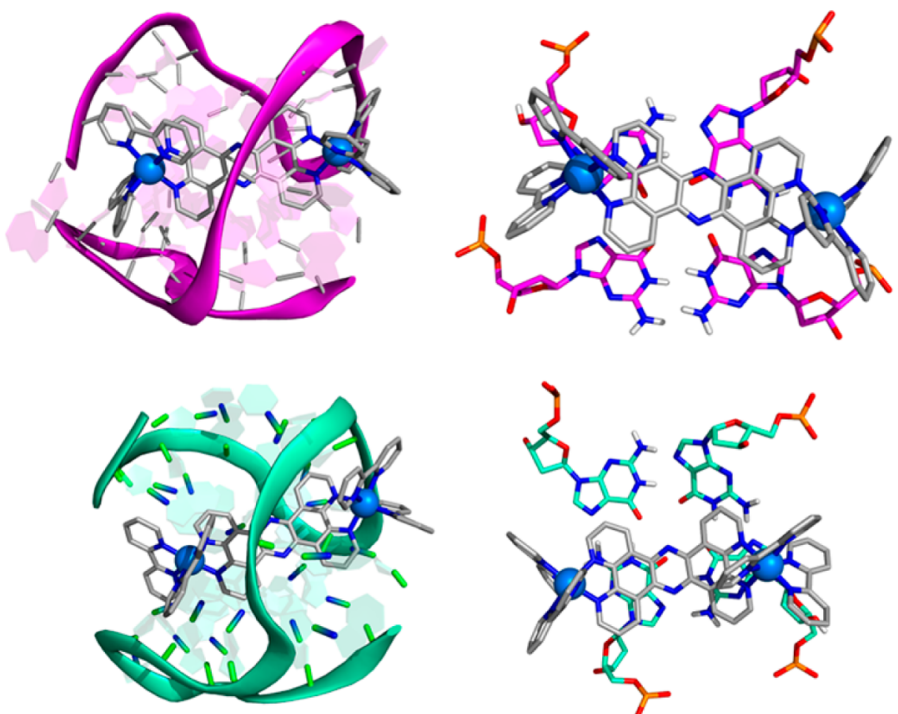

Figure 9. Representative snapshots of simulations $\boldsymbol{\Lambda} \Lambda$-1-HTS (top, magenta) and $\boldsymbol{\Delta} \boldsymbol{\Delta}$-1-HTS (bottom, aquamarine). Side and top views are presented on the left and center, respectively. Right pictures represent the complexes stacked over the top G-quartet.

tpphz bridging ligand. Thus, only the spatial arrangement of the two $\mathrm{Ru}$ (bipy) ${ }_{2}$ moieties was changed, in agreement with the respective diastereomers in the octahedral metal coordination sphere. Further molecular dynamics simulation details, together with an extended discussion, are provided in Supporting Information.

Simulations A, B, and $\mathbf{C}$ of the free HTS show that the Gtetrads yield low root mean-square deviations (RMSD), calculated relative to the starting structures, consistent with a minor conformational rearrangement experienced by these subunits throughout the $50 \mathrm{~ns}$ of simulation time, which is to be expected because they are held in place by hydrogen bonds. In contrast, the diagonal loops are more mobile (see Supporting Information Figure S06) showing higher RMSD values. Overall, the three independent simulations sampled significant HTS conformational space as suggested by the representative conformations of simulations $\mathbf{A}, \mathbf{B}$, and $\mathbf{C}$, which have slightly different structures with cross RMSD values collected in Supporting Information Table S03 between 2.71 and $3.88 \AA$. We then went on to look at the interaction of HTS with the metal complex using the same method.

Figure 8 shows the RMSD values over the simulation time (using the unrelaxed starting structures as reference) for both the $\boldsymbol{\Lambda} \mathbf{\Lambda}-\mathbf{1}-\mathbf{H T S}$ and $\boldsymbol{\Delta \Delta - 1 - H T S}$ associations. In both cases, after an initial jump due to the geometry relaxation using the ideal force field parameters, the values tend to stabilize. Focusing on the RMSD values for $\mathbf{\Lambda} \mathbf{\Lambda}-\mathbf{1}-\mathbf{H T S}$, the G-tetrad and the HTS structure typically stabilize after the first five ns while the values for the diagonal loop oscillate. This loop mobility is assigned to the presence of $\Lambda \Lambda-1$, as it was not observed in simulations A, B, and C of free HTS. Apart from this increased loop mobility, the antiparallel basket is as stable as free HTS was in simulations $\mathbf{A}, \mathbf{B}$, and $\mathbf{C}$, so unsurprisingly the RMSDs between the representative conformations of $\mathbf{\Lambda} \mathbf{\Lambda}-\mathbf{1}-\mathbf{H T S}$ and simulations $\mathbf{A}, \mathbf{B}$, and $\mathbf{C}$ are within the variability found for the calculated cross RMSDs obtained in these three simulations (see Supporting Information Table S03).

By contrast, for $\Delta \Delta-\mathbf{1}-$ HTS, all the RMSD values converge very quickly, including those associated with the diagonal loop. This indicates that complex $\Delta \Delta-1$ is able to fit under the loop without causing major G-DNA conformational changes. Indeed, the representative conformation is comparable to the representative conformation of the $\mathbf{\Lambda} \mathbf{\Lambda}$-1-HTS simulation (with a RMSD value of only $1.55 \AA$ ) and the representative frames of the free HTS simulationsm A, B, and C, with cross RMSD values ranging from 1.54 to $2.81 \AA$. This indicates that the observed experimental recognition of only $\Lambda \Lambda-1$ under the diagonal loop is not caused by the intrinsic loop cavity shape or size because both isomers are able to fit into the cavity.

Analyzing the representative conformations of $\mathbf{\Lambda} \mathbf{\Lambda}-\mathbf{1}-\mathbf{H T S}$ and $\boldsymbol{\Delta} \boldsymbol{\Delta}$-1-HTS represented in Figure 9, both ruthenium complexes bind under the diagonal loop with the tpphz ligand stacking over the top G-tetrad. However, some differences are evident. Complex $\Lambda \Lambda-1$ adopts a diagonal arrangement over 
the guanine bases and under the loop whereas in $\Delta \Delta-1$, the stacking of the tpphz ligand is more localized over two guanines of only one-half of the G-tetrad.

To clarify this point, a surface representation was constructed for the position occupied by the tpphz ligand (excluding the hydrogen atoms) over a $50 \mathrm{~ns}$ collection period for the HTS association with each of the diastereomers (Figure 10). These
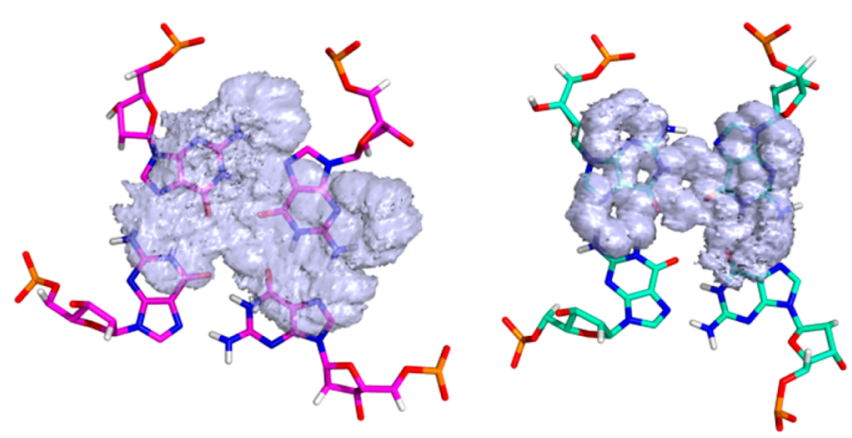

Figure 10. Surface (isovalue $=1$ ) representing the histogram of positions occupied by the tpphz atoms (excluding hydrogen) over the $50 \mathrm{~ns}$ collection period in $\mathbf{\Lambda} \mathbf{\Lambda}$-1-HTS (left) and $\boldsymbol{\Delta} \boldsymbol{\Delta}$-1-HTS (right).

surfaces clearly show a striking difference: in $\mathbf{\Lambda} \mathbf{\Lambda}-\mathbf{1}-\mathbf{H T S}$, the tpphz ligand is able to "float" over the G-tetrad interacting with the four guanines. In contrast, in $\boldsymbol{\Delta} \boldsymbol{\Delta}$-1-HTS, the tpphz ligand remains "locked" over two guanines of the G-tetrad throughout the entire simulation time. Because tpphz is present in both dinuclear complexes, the difference in dynamic behavior must be caused by the stereochemistry of the bipy ligands in the octahedral $\mathrm{Ru}$ (II) coordination spheres. This is consistent with the observation that in $\mathbf{\Lambda} \mathbf{\Lambda}-\mathbf{1}-\mathbf{H T S}$ the diagonal loop appears to be more flexible, oscillating over the middle of both $\mathrm{Ru}(\text { bipy })_{2}$ moieties. On the other hand, in $\Delta \mathbf{\Delta}$-1-HTS, the loop is more rigid and locks the $\Delta \Delta-1$ complex movement over the G-tetrad.

The above results suggest that, although both $\Lambda \Lambda-1$ and $\Delta \Delta$ 1 complexes can fit under the loop, sufficient HTS loop conformational freedom to permit the complex entrance into the prefolded G-DNA structure is only present for $\Lambda \Lambda$-1. In contrast, the stereochemistry of $\Delta \Delta-\mathbf{1}$ appears to induce increased rigidity on the loop, preventing complex entrance into the loop arch. In other words, $\Delta \Delta-\mathbf{1}$ does not bind under the diagonal loop because the loop arch does not fulfill the complex's "stereochemical requirements".

As mentioned above, the simulated annealing structure proposed for the binding of $\Delta \Delta-\mathbf{1}$ to the diagonal loop end of HTS corresponds to an impossible solution (Figure 7), which could also be rationalized by our unconstrained $\mathrm{MD}$ simulations in water. If one calculates the expected NOEs (not shown) from the representative conformations of simulations $\boldsymbol{\Lambda} \mathbf{\Lambda}$-1-HTS and $\boldsymbol{\Delta} \boldsymbol{\Delta}$-1-HTS (or from the relaxed structures obtained by Molecular Mechanics minimization), the protons that contact the ligand are quite different, meaning that the interaction of $\Lambda \Lambda-1$ and $\Delta \Delta-\mathbf{1}$ under the loop results in clearly distinct NOEs. Therefore, the experimental interactions (presumably generated only by the " $\mathbf{\Lambda} \mathbf{\Lambda}-\mathbf{1}-\mathbf{H T S}$ " interaction) are not suitable as restraints to generate a hypothetical " $\Delta \Delta-1$ HTS” association with this binding mode.

\section{CONCLUSIONS}

We have shown that $\Lambda \Lambda-2$ binds mainly at the diagonal loop end of HTS, stabilized by a good steric fit under the loop. By contrast, $\Delta \Delta-2$ binds $>40$-fold more weakly and almost entirely at the other end. Both isomers also bind to B-DNA, but the affinity of $\Lambda \Lambda-2$ is greater for HTS than for B-DNA and, importantly, when bound to HTS the luminescence is more intense and is blue-shifted from $\sim 675$ to $\sim 630 \mathrm{~nm}$. Studies with other ligands that bind to quadruplex DNA have shown that compounds that bind strongly to one conformation also stabilize that conformation, as expected from thermodynamic arguments. ${ }^{10,52,60-62}$ We have previously shown ${ }^{49,50}$ that 2 is actively taken up by cells, and confocal images show that it generates punctate images centered mainly in the heterochromatin, as might be expected for a probe that highlights Gquadruplexes. It also has a wavelength and intensity of emission that is different for different quadruplex structures. ${ }^{48} \Lambda \Lambda-2$ is therefore a useful tool for specifically stabilizing and imaging antiparallel basket structures with a diagonal loop. This study also confirms that the antiparallel structure of HTS can be selectively targeted. In comparison with the use of antibodies to detect quadruplexes, ${ }^{63-65}$ small molecules like complex 2 and its analogues are much simpler to rationally design and potentially have wider applicability as they can be used directly on living cells. Furthermore, and as this study indicates, because such systems can be made to target specific features of a quadruplex, they can be made specific to individual quadruplex structures. Consequently, with the structural information obtained by this study, we are exploring this potential for enhanced specificity through informed design of derivatives of 2. Although this work is intended to image antiparallel basket quadruplexes in living cells, it could subsequently be extended into molecular tools for selectively stabilizing quadruplexes.

\section{EXPERIMENTAL SECTION}

Ligands were synthesized using published methods. $\mathrm{Ru}(\text { phen })_{2} \mathrm{Cl}_{2}$. $2 \mathrm{H}_{2} \mathrm{O}$ and $\mathrm{Ru}$ (bipy) $)_{2} \mathrm{Cl}_{2} \cdot 2 \mathrm{H}_{2} \mathrm{O}$ were prepared following a standard literature method. ${ }^{64}$ The racemic mixtures of the tpphz complexes were prepared by methods we have previously described. ${ }^{47}$ Enantiomers of $\mathbf{2}$ were prepared by the route first described by MacDonnell and Bodige. ${ }^{66}$ Characterization data for these complexes were identical to the original reports.

The HTS oligonucleotide $\mathrm{d}\left[\mathrm{AG}_{3}\left(\mathrm{TTAG}_{3}\right)_{3}\right]$ was purchased from Eurogentec Biotechnology (Southampton, UK), purified by HPLC, and used without further annealing. Samples for UV-vis and luminescence titrations were in $10 \mathrm{mM} \mathrm{KH_{2 }} \mathrm{PO}_{4} / \mathrm{K}_{2} \mathrm{HPO}_{4}$ and 1 $\mathrm{mM} \mathrm{K} \mathrm{K}_{2} \mathrm{EDTA}$ in $50-200 \mathrm{mM} \mathrm{KCl}(\mathrm{pH} 7.0,298 \mathrm{~K}$ ), in which DNA was added to $10 \mu \mathrm{M}$ ligand, while samples for NMR were prepared in $50 \mathrm{mM} \mathrm{NaCl}, \mathrm{pH}$ 7, using $300 \mathrm{mM}$ DNA at $298 \mathrm{~K}$. Titrations were conducted with DNA and ligand of the same order of concentration as $1 / K_{\mathrm{b}}$, shown to be the optimum values for obtaining accurate binding constants. ${ }^{67}$ The optimum temperature for observing NOEs to 1 was $283 \mathrm{~K}$. CD spectra were recorded on a Jasco J-810 spectrophotometer using a Peltier variable temperature controller: $100 \mathrm{~nm} / \mathrm{min}$ from 200 to $320 \mathrm{~nm}$. CD melting experiments gave a melting temperature of $63.3^{\circ} \mathrm{C}$. Luminescence measurements were carried out on a Hitachi F4500 fluorescence spectrophotometer using a $1 \mathrm{~cm}$ path length. UVvis titrations were carried out using a Cary 3 Bio. Binding affinities were obtained by fitting using Origin 7.0 software to a standard oneset-of-binding-sites model.

NMR experiments were carried out on Bruker Avance 800, 600, and 500 spectrometers. Assignments were made using COSY, TOCSY, and NOESY spectra (mixing times 60 or $90 \mathrm{~ms}$ for TOCSY, $100 \mathrm{~ms}$ for NOESY), supported by ${ }^{1} \mathrm{H}-{ }^{31} \mathrm{P}$ HSQC (Supporting Information Table S01). Spectra were analyzed using FELIX (Felix NMR, Inc., San Diego, CA).

Structure calculations were carried out using Xplor with the parallhdg.dna parameters. Calculations used simulated annealing over 8000 steps from 2000 to $100 \mathrm{~K}$, using standard square-well potentials 
for NOEs. All calculations imposed planarity and hydrogen bonding in the tetrads plus planarity in tpphz and bipy polypyridyl ligands and octahedral geometry around the ruthenium. The force field included van der Waals and electrostatic terms. Threading of the ligand into the complex was accomplished by starting with almost zero van der Waals radii and increasing the radius in a geometric progression during the simulated annealing. For each complex, only the intermolecular NOEs relevant to that end were included, all specified as ambiguous NOEs to any ligand proton, with an upper limit of $5 \AA$. Convergence was improved by including restraints to position the tpphz ring close to the relevant tetrad plane. NOEs for the $\boldsymbol{\Lambda} \boldsymbol{\Lambda}$-1-HTS and $\boldsymbol{\Delta} \boldsymbol{\Delta}$-1-HTS complexes were calculated by numerical integration of the Solomon equations. ${ }^{68}$

Unconstrained MD simulations were carried out using the AMBER ff $99 \mathrm{bsc} 0$ set of parameters and charges for $\mathrm{DNA}^{69}$ combined with General Amber Force Field (GAFF) parameters ${ }^{70}$ with extra terms ${ }^{71,72}$ and RESP charges ${ }^{73}$ for both $\Delta \Delta-1$ and $\Lambda \Lambda-1$ complexes. These simulations were undertaken with the pmemd.cuda AMBER executable, able to accelerate explicit solvent Particle Mesh Ewald $(\mathrm{PME})^{74,75}$ calculations through the use of GPUs with the new Single Precision Fixed Point (SPFP) model. ${ }^{76}$

\section{ASSOCIATED CONTENT}

\section{S Supporting Information}

Details of MD; luminescent titrations with CT-DNA; circular dichroism spectrum of HTS quadruplex; titration with $\Delta \Delta-\mathbf{2}$; NMR assignments of the HTS quadruplex; models of $\Lambda \Lambda-2$ and $\Delta \Delta-2$ bound to HTS. This material is available free of charge via the Internet at http://pubs.acs.org.

\section{Accession Codes}

Coordinates and chemical shift data have been deposited at the $\mathrm{PDB}$ with the entry codes $2 \mathrm{mcc}(\Delta \Delta-1$ complex $)$ and $2 \mathrm{mco}$ $(\Lambda \Lambda-1$ complex $)$ and also at BMRB with the entry codes 19435 and 19448 , respectively.

\section{AUTHOR INFORMATION}

\section{Corresponding Authors}

*For J.A.T.: phone, 44114222 9325; fax, 44114222 9346; Email, james.thomas@sheffield.ac.uk.

*For M.P.W.: phone, 44114222 4224; fax, 44114222 2787; E-mail, m.williamson@sheffield.ac.uk.

*For V.F.: phone, 351234370 729; fax, 351234401 597; Email, vitor.felix@ua.pt.

Notes

The authors declare no competing financial interest.

\section{ACKNOWLEDGMENTS}

We thank the EPSRC for a Ph.D. studentship for T.W. through the White Rose Life Science DTC. P.J.C. thanks FCT for the postdoctoral grant SFRH/BPD/27082/2006. V.F. acknowledges the funding from QREN-FEDER through the Operational Program Competitiveness Factors-COMPETE and National Funds through the FCT under project PTDC/QUIQUI/101022/2008.

\section{ABBREVIATIONS USED}

HTS, human telomere sequence; tpphz, tetrapyrido[3,2-a:2',3'$\left.c: 3^{\prime \prime}, 2^{\prime \prime}-h: 2^{\prime \prime \prime}, 3^{\prime \prime \prime}-j\right]$ phenazine; bipy, 2,2'-bipyridine; phen, 1,10 -

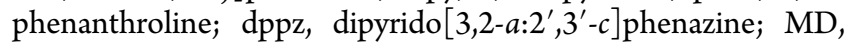
molecular dynamics; CD, circular dichroism; CT-DNA, calf thymus DNA; NOE, nuclear Overhauser effect

\section{REFERENCES}

(1) Gellert, M.; Lipsett, M. N.; Davies, D. R. Helix formation by guanylic acid. Proc. Natl. Acad. Sci. U. S. A. 1962, 48, 2013-2018.

(2) Sen, D.; Gilbert, W. Formation of parallel 4-stranded complexes by guanine-rich motifs in DNA and its implications for meiosis. Nature 1988, 334, 364-366.

(3) Paeschke, K.; Simonsson, T.; Postberg, J.; Rhodes, D.; Lipps, H. J. Telomere end-binding proteins control the formation of G-quadruplex DNA structures in vivo. Nature Struct. Mol. Biol. 2005, 12, 847-854.

(4) Kim, N. W.; Piatyszek, M. A.; Prowse, K. R.; Harley, C. B.; West, M. D.; Ho, P. L. C.; Coviello, G. M.; Wright, W. E.; Weinrich, S. L.; Shay, J. W. Specific association of human telomerase activity with immortal cells and cancer. Science 1994, 266, 2011-2015.

(5) Counter, C. M.; Hahn, W. C.; Wei, W. Y.; Caddle, S. D.; Beijersbergen, R. L.; Lansdorp, P. M.; Sedivy, J. M.; Weinberg, R. A. Dissociation among in vitro telomerase activity, telomere maintenance, and cellular immortalization. Proc. Natl. Acad. Sci. U. S. A. 1998, 95, $14723-14728$.

(6) Zahler, A. M.; Williamson, J. R; Cech, T. R.; Prescott, D. M. Inhibition of telomerase by G-quartet DNA structures. Nature 1991, 350, 718-720.

(7) Huppert, J. L.; Balasubramanian, S. G-Quadruplexes in promoters throughout the human genome. Nucleic Acids Res. 2007, 35, 406-413.

(8) Verma, A.; Halder, K.; Halder, R.; Yadav, V. K.; Rawal, P.; Thakur, R. K.; Mohd, F.; Sharma, A.; Chowdhury, S. Genome-wide computational and expression analyses reveal G-quadruplex DNA motifs as conserved cis-regulatory elements in human and related species. J. Med. Chem. 2008, 51, 5641-5649.

(9) Du, Z.; Zhao, Y.; Li, N. Genome-wide analysis reveals regulatory role of G4 DNA in gene transcription. Genome Res. 2008, 18, 233241.

(10) Siddiqui-Jain, A.; Grand, C. L.; Bearss, D. J.; Hurley, L. H. Direct evidence for a G-quadruplex in a promoter region and its targeting with a small molecule to repress c-MYC transcription. Proc. Natl. Acad. Sci. U. S. A. 2002, 99, 11593-11598.

(11) Dai, J. X.; Dexheimer, T. S.; Chen, D.; Carver, M.; Ambrus, A.; Jones, R. A.; Yang, D. Z. An intramolecular G-quadruplex structure with mixed parallel/antiparallel G-strands formed in the human BCL-2 promoter region in solution. J. Am. Chem. Soc. 2006, 128, 1096-1098.

(12) Cogoi, S.; Xodo, L. E. G-quadruplex formation within the promoter of the KRAS proto-oncogene and its effect on transcription. Nucleic Acids Res. 2006, 34, 2536-2549.

(13) Shirude, P. S.; Okumus, B.; Ying, L.; Ha, T.; Balasubramanian, S. Single-molecule conformational analysis of G-quadruplex formation in the promoter DNA duplex of the proto-oncogene C-kit. J. Am. Chem. Soc. 2007, 129, 7484-7485.

(14) Kumari, S.; Bugaut, A.; Huppert, J. L.; Balasubramanian, S. An RNA G-quadruplex in the 5 ' UTR of the NRAS proto-oncogene modulates translation. Nature Chem. Biol. 2007, 3, 218-221.

(15) Haider, S. M.; Neidle, S.; Parkinson, G. N. A structural analysis of G-quadruplex/ligand interactions. Biochimie 2011, 93, 1239-1251.

(16) Xue, Y.; Kan, Z.-Y.; Wang, Q.; Yao, Y.; Liu, J.; Hao, Y.-H.; Tan, Z. Human telomeric DNA forms parallel-stranded intramolecular Gquadruplex in $\mathrm{K}+$ solution under molecular crowding condition. J. Am. Chem. Soc. 2007, 129, 11185-11191.

(17) Heddi, B.; Anh Tuan, P. Structure of human telomeric DNA in crowded solution. J. Am. Chem. Soc. 2011, 133, 9824-9833.

(18) Dhakal, S.; Cui, Y.; Koirala, D.; Ghimire, C.; Kushwaha, S.; Yu, Z.; Yangyuoru, P. M.; Mao, H. Structural and mechanical properties of individual human telomeric G-quadruplexes in molecularly crowded solutions. Nucleic Acids Res. 2013, 41, 3915-3923.

(19) Miller, M. C.; Buscaglia, R.; Chaires, J. B.; Lane, A. N.; Trent, J. $\mathrm{O}$. Hydration is a major determinant of the G-quadruplex stability and conformation of the human telomere 3 ' sequence of $\mathrm{d}$ $\left(\mathrm{AG}_{3}\left(\mathrm{TTAG}_{3}\right)_{3}\right)$. J. Am. Chem. Soc. 2010, 132, 17105-17107.

(20) Wang, Y.; Patel, D. J. Solution structure of the human telomeric repeat $\mathrm{d}\left[\mathrm{AG}_{3}\left(\mathrm{~T}_{2} \mathrm{AG}_{3}\right)_{3}\right]$ G-tetraplex. Structure 1993, 1, 263-282. 
(21) Parkinson, G. N.; Lee, M. P. H.; Neidle, S. Crystal structure of parallel quadruplexes from human telomeric DNA. Nature 2002, 417, 876-880.

(22) Ambrus, A.; Chen, D.; Dai, J. X.; Bialis, T.; Jones, R. A.; Yang, D. Z. Human telomeric sequence forms a hybrid-type intramolecular Gquadruplex structure with mixed parallel/antiparallel strands in potassium solution. Nucleic Acids Res. 2006, 34, 2723-2735.

(23) Luu, K. N.; Phan, A. T.; Kuryavyi, V.; Lacroix, L.; Patel, D. J. Structure of the human telomere in $\mathrm{K}^{+}$solution: an intramolecular (3 + 1) G-quadruplex scaffold. J. Am. Chem. Soc. 2006, 128, 9963-9970. (24) Dai, J.; Carver, M.; Punchihewa, C.; Jones, R. A.; Yang, D. Structure of the hybrid-2 type intramolecular human telomeric Gquadruplex in $\mathrm{K}^{+}$solution: insights into structure polymorphism of the human telomeric sequence. Nucleic Acids Res. 2007, 35, 4927-4940.

(25) Lim, K. W.; Amrane, S.; Bouaziz, S.; Xu, W.; Mu, Y.; Patel, D. J.; Luu, K. N.; Phan, A. T. Structure of the human telomere in $\mathrm{K}^{+}$ solution: a stable basket-type G-quadruplex with only two G-tetrad layers. J. Am. Chem. Soc. 2009, 131, 4301-4309.

(26) Renčiuk, D.; Kejnovská, I.; Školáková, P.; Bednářová, K.; Motlová, J.; Vorlíčková, M. Arrangements of human telomere DNA quadruplex in physiologically relevant $\mathrm{K}^{+}$solutions. Nucleic Acids Res. 2009, 37, 6625-6634.

(27) Hänsel, R.; Löhr, F.; Foldynová-Trantírková, S.; Bamberg, E.; Trantírek, L.; Dötsch, V. The parallel G-quadruplex structure of vertebrate telomeric repeat sequences is not the preferred folding topology under physiological conditions. Nucleic Acids Res. 2011, 39, 5768-5775.

(28) Neidle, S. The structures of quadruplex nucleic acids and their drug complexes. Curr. Opin. Struct. Biol. 2009, 19, 239-250.

(29) Georgiades, S. N.; Abd Karim, N. H.; Suntharalingam, K.; Vilar, R. Interaction of metal complexes with G-quadruplex DNA. Angew. Chem., Int. Ed. 2010, 49, 4020-4034.

(30) Ou, T.-M.; Lu, Y.-J.; Tan, J.-H.; Huang, Z.-S.; Wong, K.-Y.; Gu, L.-Q. G-Quadruplexes: targets in anticancer drug design. ChemMedChem 2008, 3, 690-713.

(31) $\mathrm{Xu}, \mathrm{Y}$. Chemistry in human telomere biology: structure, function and targeting of telomere DNA/RNA. Chem. Soc. Rev. 2011, 40, 2719-2740.

(32) Campbell, N. H.; Abd Karim, N. H.; Parkinson, G. N.; Gunaratnam, M.; Petrucci, V.; Todd, A. K.; Vilar, R.; Neidle, S. Molecular basis of structure-activity relationships between salphen metal complexes and human telomeric DNA quadruplexes. J. Med. Chem. 2012, 55, 209-222.

(33) Parkinson, G. N.; Cuenca, F.; Neidle, S. Topology conservation and loop flexibility in quadruplex-drug recognition: Crystal structures of inter- and intramolecular telomeric DNA quadruplex-drug complexes. J. Mol. Biol. 2008, 381, 1145-1156.

(34) Bazzicalupi, C.; Ferraroni, M.; Bilia, A. R.; Scheggi, F.; Gratteri, P. The crystal structure of human telomeric DNA complexed with berberine: an interesting case of stacked ligand to G-tetrad ratio higher than 1:1. Nucleic Acids Res. 2013, 41, 632-638.

(35) Collie, G. W.; Promontorio, R.; Hampel, S. M.; Micco, M.; Neidle, S.; Parkinson, G. N. Structural basis for telomeric Gquadruplex targeting by naphthalene diimide ligands. J. Am. Chem. Soc. 2012, 134, 2723-2731.

(36) Micco, M.; Collie, G. W.; Dale, A. G.; Ohnmacht, S. A.; Pazitna, I.; Gunaratnam, M.; Reszka, A. P.; Neidle, S. Structure-based design and evaluation of naphthalene diimide G-quadruplex ligands as elomere targeting agents in pancreatic cancer cells. J. Med. Chem. 2013, 56, 2959-2974.

(37) Fedoroff, O. Y.; Salazar, M.; Han, H. Y.; Chemeris, V. V.; Kerwin, S. M.; Hurley, L. H. NMR-based model of a telomeraseinhibiting compound bound to G-quadruplex DNA. Biochemistry 1998, 37, 12367-12374.

(38) Gavathiotis, E.; Heald, R. A.; Stevens, M. F. G.; Searle, M. S. Drug recognition and stabilisation of the parallel-stranded DNA quadruplex $\mathrm{d}$ (TTAGGGT) ${ }_{4}$ containing the human telomeric repeat. $J$. Mol. Biol. 2003, 334, 25-36.
(39) Clark, G. R.; Pytel, P. D.; Squire, C. J.; Neidle, S. Structure of the first parallel DNA quadruplex-drug complex. J. Am. Chem. Soc. 2003, 125, 4066-4067.

(40) Parkinson, G. N.; Ghosh, R.; Neidle, S. Structural basis for binding of porphyrin to human telomeres. Biochemistry 2007, 46, $2390-2397$.

(41) Martino, L.; Virno, A.; Pagano, B.; Virgilio, A.; Di Micco, S.; Galeone, A.; Giancola, C.; Bifulco, G.; Mayol, L.; Randazzo, A. Structural and thermodynamic studies of the interaction of distamycin a with the parallel quadruplex structure $[\mathrm{d}(\mathrm{TGGGGT})]_{4}$. J. Am. Chem. Soc. 2007, 129, 16048-16056.

(42) Haider, S. M.; Parkinson, G. N.; Neidle, S. Structure of a Gquadruplex-ligand complex. J. Mol. Biol. 2003, 326, 117-125.

(43) Campbell, N. H.; Patel, M.; Tofa, A. B.; Ghosh, R.; Parkinson, G. N.; Neidle, S. Selectivity in ligand recognition of G-quadruplex loops. Biochemistry 2009, 48, 1675-1680.

(44) Metcalfe, C.; Thomas, J. A. Kinetically inert transition metal complexes that reversibly bind to DNA. Chem. Soc. Rev. 2003, 32, 215-224.

(45) Gill, M. R.; Thomas, J. A. Ruthenium(II) polypyridyl complexes and DNA-from structural probes to cellular imaging and therapeutics. Chem. Soc. Rev. 2012, 41, 3179-3192.

(46) Waywell, P.; Gonzalez, V.; Gill, M. R.; Adams, H.; Meijer, A. J. H. M.; Williamson, M. P.; Thomas, J. A. Structure of the complex of $[\mathrm{Ru}(\mathrm{tpm})(\mathrm{dppz}) \mathrm{py}]^{2+}$ with a B-DNA oligonucleotide: a singlesubstituent binding switch for a metallo-intercalator. Chem.-Eur. J. 2010, 16, 2407-2417.

(47) Rajput, C.; Rutkaite, R.; Swanson, L.; Haq, I.; Thomas, J. A. Dinuclear monointercalating Ru-II complexes that display high affinity binding to duplex and quadruplex DNA. Chem.-Eur. J. 2006, 12, 4611-4619.

(48) Wilson, T.; Williamson, M. P.; Thomas, J. A. Differentiating quadruplexes: binding preferences of a luminescent dinuclear ruthenium(II) complex with four-stranded DNA structures. Org. Biomol. Chem. 2010, 8, 2617-2621.

(49) Gill, M. R.; Garcia-Lara, J.; Foster, S. J.; Smythe, C.; Battaglia, G.; Thomas, J. A. A ruthenium(II) polypyridyl complex for direct imaging of DNA structure in living cells. Nature Chem. 2009, 1, 662667.

(50) Tian, X.; Gill, M. R.; Canton, I.; Thomas, J. A.; Battaglia, G. Live cell luminescence imaging as a function of delivery mechanism. ChemBioChem 2011, 12, 548-551.

(51) Yu, H.; Wang, X.; Fu, M.; Ren, J.; Qu, X. Chiral metallosupramolecular complexes selectively recognize human telomeric Gquadruplex DNA. Nucleic Acids Res. 2008, 36, 5695-5703.

(52) Zhao, C.; Geng, J.; Feng, L.; Ren, J.; Qu, X. Chiral metallosupramolecular complexes selectively induce human telomeric Gquadruplex formation under salt-deficient conditions. Chem.-Eur. J. 2011, 17, 8209-8215.

(53) Shinohara, K.; Sannohe, Y.; Kaieda, S.; Tanaka, K.; Osuga, H.; Tahara, H.; Xu, Y.; Kawase, T.; Bando, T.; Sugiyama, H. A chiral wedge molecule inhibits telomerase activity. J. Am. Chem. Soc. 2010, 132, 3778-3782.

(54) Andersson, J.; Lincoln, P. Stereoselectivity for DNA threading intercalation of short binuclear ruthenium complexes. J. Phys. Chem. B 2011, 115, 14768-14775.

(55) Johansson, J. R.; Wang, Y.; Eng, M. P.; Kann, N.; Lincoln, P.; Andersson, J. Bridging ligand length controls AT selectivity and enantioselectivity of binuclear ruthenium threading intercalators. Chem.-Eur. J. 2013, 19, 6246-6256.

(56) Hall, J. P.; O’Sullivan, K.; Naseer, A.; Smith, J. A.; Kelly, J. M.; Cardin, C. J. Structure determination of an intercalating ruthenium dipyridophenazine complex which kinks DNA by semiintercalation of a tetraazaphenanthrene ligand. Proc. Natl. Acad. Sci. U. S. A. 2011, 108, 17610-17614.

(57) Niyazi, H.; Hall, J. P.; O’Sullivan, K.; Winter, G.; Sorensen, T.; Kelly, J. M.; Cardin, C. J. Crystal structures of $\Lambda$-Ru(phen $)_{2} \mathrm{dppz}^{2+}$ with oligonucleotides containing TA/TA and AT/AT steps show two intercalation modes. Nature Chem. 2012, 4, 621-628. 
(58) Song, H.; Kaiser, J. T.; Barton, J. K. Crystal structure of $\Delta$ $\mathrm{Ru}(\mathrm{bpy})_{2} \mathrm{dppz}^{2+}$ bound to mismatched DNA reveals side-by-side metalloinsertion and intercalation. Nature Chem. 2012, 4, 615-620.

(59) Wu, L.; Reymer, A.; Persson, C.; Kazimierczuk, K.; Brown, T.; Lincoln, P.; Nordén, B.; Billeter, M. Initial DNA interactions of the binuclear threading intercalator $\Lambda, \Lambda$ - $\left[m\right.$-bidppz (bipy) ${ }_{4} \mathrm{Ru}_{2}{ }^{4+}$ : an NMR study with d(CGCGAATTCGCG) ${ }_{2}$. Chem.-Eur. J. 2013, 19, 54015410.

(60) Seenisamy, J.; Rezler, E. M.; Powell, T. J.; Tye, D.; Gokhale, V.; Joshi, C. S.; Siddiqui-Jain, A.; Hurley, L. H. The dynamic character of the G-quadruplex element in the c-MYC promoter and modification by TMPyP4. J. Am. Chem. Soc. 2004, 126, 8702-8709.

(61) Shi, S.; Geng, X. T.; Zhao, J.; Yao, T. M.; Wang, C. R.; Yang, D. J.; Zheng, L. F.; Ji, L. N. Interaction of $\left[\mathrm{Ru}(\mathrm{bpy})_{2}(\mathrm{dppz})\right]^{2+}$ with human telomeric DNA: preferential binding to G-quadruplexes over imotif. Biochimie 2010, 92, 370-377.

(62) Sun, D.; Liu, Y.; Liu, D.; Zhang, R.; Yang, X.; Liu, J. Stabilization of G-quadruplex DNA, inhibition of telomerase activity and live cell imaging studies of chiral ruthenium(II) complexes. Chem.-Eur. J. 2012, 18, 4285-4295.

(63) Biffi, G.; Tannahill, D.; McCafferty, J.; Balasubramanian, S. Quantitative visualization of DNA G-quadruplex structures in human cells. Nature Chem. 2013, 5, 182-186.

(64) Schaffitzel, C.; Berger, I.; Postberg, J.; Hanes, J.; Lipps, H. J.; Pluckthun, A. In vitro generated antibodies specific for telomeric guanine-quadruplex DNA react with Stylonychia lemnae macronuclei. Proc. Natl. Acad. Sci. U. S. A. 2001, 98, 8572-8577.

(65) Sullivan, B. P.; Salmon, D. J.; Meyer, T. J. Mixed phosphine 2,2'bipyridine complexes of ruthenium. Inorg. Chem. 1978, 17, 33343341.

(66) MacDonnell, F. M.; Bodige, S. Efficient stereospecific syntheses of chiral ruthenium dimers. Inorg. Chem. 1996, 35, 5758-\&.

(67) Granot, J. Determination of dissociation constants of $1: 1$ complexes from NMR data. Optimization of the experimental setup by statistical analysis of simulated experiments. J. Magn. Reson. 1983, 55, 216-224.

(68) Williamson, M. P. Guidelines for the design of kinetic NOE experiments from computer simulation. Magn. Reson. Chem. 1987, 25, $356-361$.

(69) Perez, A.; Marchan, I.; Svozil, D.; Sponer, J.; Cheatham, T. E., III; Laughton, C. A.; Orozco, M. Refinenement of the AMBER force field for nucleic acids: Improving the description of a/g conformers. Biophys. J. 2007, 92, 3817-3829.

(70) Wang, J. M.; Wolf, R. M.; Caldwell, J. W.; Kollman, P. A.; Case, D. A. Development and testing of a general Amber force field. J. Comput. Chem. 2004, 25, 1157-1174.

(71) Brandt, P.; Norrby, T.; Akermark, E.; Norrby, P. O. Molecular mechanics (MM3*) parameters for ruthenium(II)-polypyridyl complexes. Inorg. Chem. 1998, 37, 4120-4127.

(72) Moret, M.-E.; Tavernelli, I.; Rothlisberger, U. Combined QM/ $\mathrm{MM}$ and classical molecular dynamics study of $\mathrm{Ru}(\text { bpy })_{3}{ }^{2+}$ in water. $J$. Phys. Chem. B 2009, 113, 7737-7744.

(73) Bayly, C. I.; Cieplak, P.; Cornell, W. D.; Kollman, P. A. A wellbehaved electrostatic potential based method using charge restraints for deriving atomic charges: the RESP model. J. Phys. Chem. 1993, 97, 10269-10280

(74) Darden, T.; York, D.; Pedersen, L. Particle mesh Ewald: an $N \cdot \log (N)$ method for Ewald sums in large systems. J. Chem. Phys. 1993, 98, 10089-10092.

(75) Essmann, U.; Perera, L.; Berkowitz, M. L.; Darden, T.; Lee, H.; Pedersen, L. G. A smooth particle mesh Ewald method. J. Chem. Phys. 1995, 103, 8577-8593.

(76) Le Grand, S.; Goetz, A. W.; Walker, R. C. SPFP: Speed without compromise: a mixed precision model for GPU accelerated molecular dynamics simulations. Comput. Phys. Commun. 2013, 184, 374-380. 